V.R. Shaginyan · A.Z. Msezane · V.A.

Stephanovich · K.G. Popov · G.S. Japaridze

\title{
Universal behavior of quantum spin liquid and optical conductivity in the insulator herbertsmithite
}

the date of receipt and acceptance should be inserted later

\begin{abstract}
We analyze optical conductivity with the goal to demonstrate experimental manifestation of a new state of matter, the so-called fermion condensate. Fermion condensates are realized in quantum spin liquids, exhibiting typical behavior of heavy fermion metals. Measurements of the low-frequency optical conductivity collected on the geometrically frustrated insulator herbertsmithite provide important experimental evidence of the nature of its quantum spin liquid composed of spinons. To analyze recent measurements of the herbertsmithite optical conductivity at different temperatures, we employ a model of strongly correlated quantum spin liquid located near the fermion condensation phase transition. Our theoretical analysis of the optical conductivity allows us to expose the physical mechanism of its temperature dependence. We also predict a dependence of the optical conductivity on a magnetic field. We consider an experimental manifestation (optical conductivity) of a new state of matter (so-called fermion condensate) realized in quantum spin liquids, for, in many ways, they exhibit typical behavior of heavy-fermion metals. Measurements of the low-frequency optical conductivity
\end{abstract}

V.R. Shaginyan

Petersburg Nuclear Physics Institute RAS;

Gatchina, 188300, Russia,

CTSPS, Clark Atlanta University,

Atlanta, Georgia 30314, USA

E-mail: vrshag@ @thd.pnpi.spb.ru

A.Z. Msezane

CTSPS, Clark Atlanta University,

Atlanta, Georgia 30314, USA

V.A. Stephanovich

Institute of Physics, Opole University,

Oleska 48, 45-052, Opole, Poland

E-mail: stef@uni.opole.pl

K.G.Popov

Komi Science Center, Ural Division, RAS, 3a, Chernova str. Syktyvkar, 167982, Russia

G. S. Japaridze

Clark Atlanta University, Atlanta, GA 30314, USA 
collected on the geometrically frustrated insulator herbertsmithite produce important experimental evidence of the nature of its quantum spin liquid composed of spinons. To analyze recent measurements of the herbertsmithite optical conductivity at different temperatures, we employ a model of strongly correlated quantum spin liquid located near the fermion condensation phase transition. Our theoretical analysis of the optical conductivity allows us to reveal the physical mechanism of its temperature dependence. We also predict a dependence of the optical conductivity on a magnetic field.

Keywords quantum phase transition, flat bands, non-Fermi-liquid states, optical conductivity, strongly correlated Fermi systems, quantum spin liquids

\section{Introduction}

The herbertsmithite $\mathrm{ZnCu}_{3}(\mathrm{OH})_{6} \mathrm{Cl}_{2}$ has a two-dimensional (2D) triangular lattice with the geometric frustration prohibiting the formation of spin ordering even at the lowest accessible temperatures $T$, and has been interpreted as a $S=1 / 2$ kagome antiferromagnet [1-5]. Magnetic kagome planes consisting of $\mathrm{Cu}^{2+}$ ions having spins $S=1 / 2$ are separated by a nonmagnetic $\mathrm{Zn}^{2+}$ layers. Observations have found no evidence of a long-range magnetic order or spin freezing down to temperature of $50 \mathrm{mK}$, indicating that $\mathrm{ZnCu}_{3}(\mathrm{OH})_{6} \mathrm{Cl}_{2}$ is the most optimal compound found to hold the quantum spin liquid (QSL) [1, 3-5]. These results are confirmed by theoretical considerations demonstrating that the ground state of kagome antiferromagnet is a gapless strongly correlated quantum spin liquid (SCQSL) [6-10]. Therefore, the above insulator properties offer unique insight into the physics of QSL. Indeed, measurements of the heat capacity reveal a $T$-linear term indicating that the low-energy excitation spectrum from the ground state is gapless $[1,3,4]$. The excitation spectrum can be obtained from the low-temperature measurements of the heat conductivity $\kappa(T)$. For instance, at $T \rightarrow 0$ residual value in $\kappa / T$ signals that the excitation spectrum is gapless. The presence of the residual value would clearly confirm the presence of QSL in $\mathrm{ZnCu}_{3}(\mathrm{OH})_{6} \mathrm{Cl}_{2}$, while the $\kappa / T \rightarrow 0$ behavior suggests that the low-energy excitation spectrum could have a gap [11]. The heat conductivity is formed primarily by both acoustic phonons and itinerant spinons, while the latter form QSL. Since the phonon contribution is negligible to the applied magnetic field $B$, the elementary excitations of QSL can be further explored by the magnetic field dependence of $k$. Since the variation of the thermal conductivity, measured under the application of magnetic field, probes elementary itinerant excitations and is insensitive to phonon's contributions, which "contaminate" $\kappa$, we suggest that measurements of $\kappa(B)$ should shed light on the nature of the ground state of QSL of herbertsmithite [11].

Measurements of the real part of the low-frequency optical conductivity $\bar{\sigma}$ as a function of the temperature $T$ and the applied of magnetic field $B$ collected on insulators with geometrical frustration produce important experimental results regarding the nature of quantum spin liquid composed of spinons [12,13]. Therefore we face a challenging problem of interpreting the experimental data [12] in a consistent way, including the $T$-dependence of the conductivity. 
In this paper we show that SCQSL represents the new state of matter, and employ the model of SCQSL to explain the observed value of the optical conductivity $\bar{\sigma}$ and its temperature dependence in herbertsmithite. We demonstrate that SCQSL is located near the topological fermion condensation quantum phase transition (FCQPT), and predict a magnetic field $B$ dependence of $\bar{\sigma}$. Our calculations are in a good agreement with experimental data.

\section{Strongly correlated quantum spin liquids}

Strongly correlated quantum spin liquids represent a special case of ordinary QSL, being a quantum state of matter composed of spinons - chargeless fermionic quasiparticles having spin $1 / 2[7,9,11,14]$. In the case of an ideal two-dimensional lattice of insulating compounds, SCQSL can emerge provided that the geometrical frustration of the lattice leads to a dispersionless topologically protected branch of the spectrum with the zero excitation energy known as the flat band [15-19]. Then, FCQPT can be considered as quantum critical point of SCQSL, composed of chargeless heavy spinons with $S=1 / 2$ and the effective mass $M^{*}$, occupying the corresponding Fermi sphere with the Fermi momentum $p_{F}$. Consequently, the properties of insulating compounds coincide with those of heavy-fermion metals with one exception. Namely, the typical insulating compound resists the flow of the electric charge [7-9]. As we are dealing with compounds having non-ideal triangular and kagome lattices, we have to bear in mind that the magnetic interactions, impurities and possible distortion of the lattices can shift the SCQSL from the exact FCQPT, positioning it somewhere near it. Therefore, the actual location of the SCQSL with respect to FCQPT has to be established solely from experimental data analysis.

The usual approach to describe the systems with itinerant fermions is the famous Landau Fermi liquid (LFL) theory [20]. The LFL theory is based on the mapping of the strongly interacting system of real electrons and nuclei in a solid to that of a weakly interacting Fermi gas. This implies that the elementary excitations behave as corresponding quasiparticles, determining the physical properties of the system at low temperatures. These quasiparticle excitations have a certain effective mass $M^{*}$, which is almost independent of temperature, pressure, and magnetic field strength being a parameter of the theory [20-22]. The LFL theory fails to explain the experimental results related to the dependence of $M^{*}$ on the temperature $T$, magnetic field $B$, pressure and other external parameters; in the vicinity of the FCQPT, deviations from the LFL behavior are observed [6]. These so-called non-Fermi-liquid (NFL) anomalies are generated by large value of the effective mass $M^{*}$ associated with FCQPT.

To make our paper self-contained, we begin with a brief outline of the physical mechanism, responsible for the dependence of the Landau quasiparticle effective mass, $M^{*}(B, T)$, on magnetic field and temperature (we recollect that in LFL theory the effective mass does not strongly depend on external parameters). The key point here is that the effective mass begins to depend strongly on temperature $T$, magnetic field $B$ and other external parameters such as the pressure $P$ near the above FCQPT [6]. The main feature of our approach is based on the existence of one more instability channel for the Landau Fermi liquid (in addition to the wellknown Pomeranchuk instability channel, see e.g. Ref. [21]). Namely, under some 
conditions (see Ref. [14] for details) the effective mass of a Landau quasiparticle may become infinite. In this case, to avoid unphysical situations related to either effective mass divergence or even its negativity, the system alters its Fermi surface topology so that the effective mass acquires temperature and magnetic field dependencies. To investigate the low temperature transport properties, the scaling behavior, and the effective mass $M^{*}(B, T)$ of SCQSL, we use the model of homogeneous heavy fermion liquid. In that case, the model allows avoidance of complications associated with the crystalline anisotropy of solids [6], and the Landau equation, describing the effective mass $M^{*}$ of a heavy fermion liquid, reads $[6,20]$

$$
\begin{aligned}
\frac{1}{M^{*}(B, T)} & =\frac{1}{M}+\sum_{\sigma_{1}} \int \frac{\mathbf{p}_{F} \mathbf{p}}{p_{F}^{3}} F\left(\mathbf{p}_{\mathbf{F}}, \mathbf{p}\right) \\
& \times \frac{\partial n_{\sigma_{1}}(\mathbf{p}, T, B)}{\partial p} \frac{d \mathbf{p}}{(2 \pi)^{3}},
\end{aligned}
$$

where $M$ is the corresponding bare mass, $F\left(\mathbf{p}_{\mathbf{F}}, \mathbf{p}\right)$ is the Landau interaction function, which depends on Fermi momentum $p_{F}$, momentum $p$, with $F\left(\mathbf{p}_{\mathbf{F}}, \mathbf{p}\right)$ is phenomenological function, obtained from the condition of the best fit to experiment, and $\sigma$ is the spin index. For simplicity, we assume that the Landau interaction does not depend on the spins. At finite temperatures, the distribution function $n$ can be expressed as

$$
n_{\sigma}(\mathbf{p}, T)=\left\{1+\exp \left[\frac{\left(\varepsilon_{\sigma}(\mathbf{p}, T)-\mu_{\sigma}\right)}{T}\right]\right\}^{-1}
$$

where $\varepsilon_{\sigma}(\mathbf{p}, T)$ is the single-particle spectrum. In our case, the chemical potential $\mu$ depends on the spin due to Zeeman splitting $\mu_{\sigma}=\mu \pm \mu_{B} B, \mu_{B}$ is the Bohr magneton.

In LFL theory, the single-particle spectrum is a variational derivative of the system energy $E\left[n_{\sigma}(\mathbf{p}, T)\right]$ with respect to occupation number $n$,

$$
\varepsilon_{\sigma}(\mathbf{p}, T)=\frac{\delta E[n(\mathbf{p})]}{\delta n_{\sigma}} .
$$

Choice of the interaction function shape and parameters is dictated to by the fact that the system has to be at FCQPT [6, 23, 24]. Thus, the sole role of the Landau interaction is to bring the system to the FCQPT point, where the Fermi surface alters its topology so that the effective mass acquires temperature and field dependence [6,23]. The variational procedure, applied to the functional $E\left[n_{\sigma}(\mathbf{p}, T)\right]$, gives the following explicit form for $\varepsilon_{\sigma}(\mathbf{p}, T)$,

$$
\frac{\partial \varepsilon_{\sigma}(\mathbf{p}, T)}{\partial \mathbf{p}}=\frac{\mathbf{p}}{M}-\int \frac{\partial F\left(\mathbf{p}, \mathbf{p}_{1}\right)}{\partial \mathbf{p}} n_{\sigma}\left(\mathbf{p}_{1}, T\right) \frac{d^{3} p_{1}}{(2 \pi)^{3}},
$$

Equations (2) and (3) constitute the closed set for self-consistent determination of $\varepsilon_{\sigma}(\mathbf{p}, T)$ and $n_{\sigma}(\mathbf{p}, T)$ and the effective mass, $p_{F} / M^{*}=\partial \varepsilon(p) /\left.\partial p\right|_{p=p_{F}}$. We emphasize here that in our approach the temperature and the magnetic field dependence of the effective mass is derived solely from the $T$ and the $B$ dependence 
of $\varepsilon_{\sigma}(\mathbf{p})$ and $n_{\sigma}(\mathbf{p})$. At the point of FCQPT, Eq. (1) can be solved analytically $[6,23]$. At $B=0$, the effective mass strongly depends on $T$, demonstrating the NFL behavior

$$
M^{*}(T) \simeq a_{T} T^{-2 / 3} .
$$

At finite $T$, the application of magnetic field $B$ drives the system the to LFL region with

$$
M^{*}(B) \simeq a_{B} B^{-2 / 3} .
$$

A deeper insight into the behavior of $M^{*}(B, T)$ can be achieved using some "internal" (or natural) scales. Namely, near FCQPT the solution $M^{*}(B, T)$ of Eq. (1) reaches its maximal value $M_{M}^{*}$ at certain temperature $T_{M} \propto B$ [6]. Hence, it is convenient to introduce the internal scales $M_{M}^{*}$ and $T_{M}$ to measure the effective mass and temperature respectively. In other words, we divide the mass and temperature by $M_{M}^{*}$ and $T_{M}$. This generates the normalized effective mass $M_{N}^{*}=M^{*} / M_{M}^{*}$ and the temperature $T_{N}=T / T_{M}$. Near FCQPT the normalized solution of Eq. (1) $M_{N}^{*}\left(T_{N}\right)$ can be well approximated by a simple universal interpolating function [6]. The interpolation occurs between the LFL and NFL regimes and represents the universal scaling behavior of $M_{N}^{*}[6]$

$$
M_{N}^{*}(y) \approx c_{0} \frac{1+c_{1} y^{2}}{1+c_{2} y^{8 / 3}} .
$$

Here, $y=T_{N}=T / T_{M}, c_{0}=\left(1+c_{2}\right) /\left(1+c_{1}\right)$, and $c_{1}, c_{2}$ are fitting parameters. The magnetic field $B$ enters Eq. (1) only in the combination $\mu_{B} B / T$, making $T_{M} \sim \mu_{B} B$. It follows from Eq. (6) that

$$
T_{M} \simeq a_{1} \mu_{B} B,
$$

where $a_{1}$ is a dimensionless factor, $\mu_{B}$ is the Bohr magneton. Thus, in the presence of magnetic field the variable $y$ becomes $y=T / T_{M} \sim T / \mu_{B} B$. Taking Eq. (7) into account, we conclude that Eq. (6) describes the scaling behavior of the effective mass as a function of $T$ and $B$ : The curves $M_{N}^{*}$ at different magnetic fields $B$ merge into a single one in terms of the normalized variable $y=T / T_{M}$. Since the variables $T$ and $B$ enter symmetrically, Eq. (6) also describes the scaling behavior of $M_{N}^{*}(B, T)$ as a function of $B$ at fixed $T$.

Now we construct the schematic phase diagram of SCQSL of the insulator herbertsmithite. The phase diagram is reported in Fig. 1. We assume for simplicity that at $T=0$ and $B=0$ the system is approximately located at FCQPT without tuning. Both magnetic field $B$ and temperature $T$ play the role of the control parameters, shifting the system from FCQPT and driving it from the NFL to LFL regions as shown by the vertical and horizontal arrows. At fixed temperatures the increase of $B$ drives the system along the horizontal arrow from the NFL region to LFL one. At the fixed magnetic field and increasing temperatures the system transits along the vertical arrow from the LFL region to the NFL one. The hatched area denoting the transition region separates the NFL state from the weakly polarized LFL one. The transition temperature $T^{*}(B)$ is given by

$$
T^{*}(B) \simeq T_{M}(B),
$$

which directly follows from Eq. (7) ( $a_{2}$ is a dimensionless factor). The solid line represents the transition region, $T^{*}(B) \simeq T_{M}(B)$. Referring to Eq. (8), this line is 


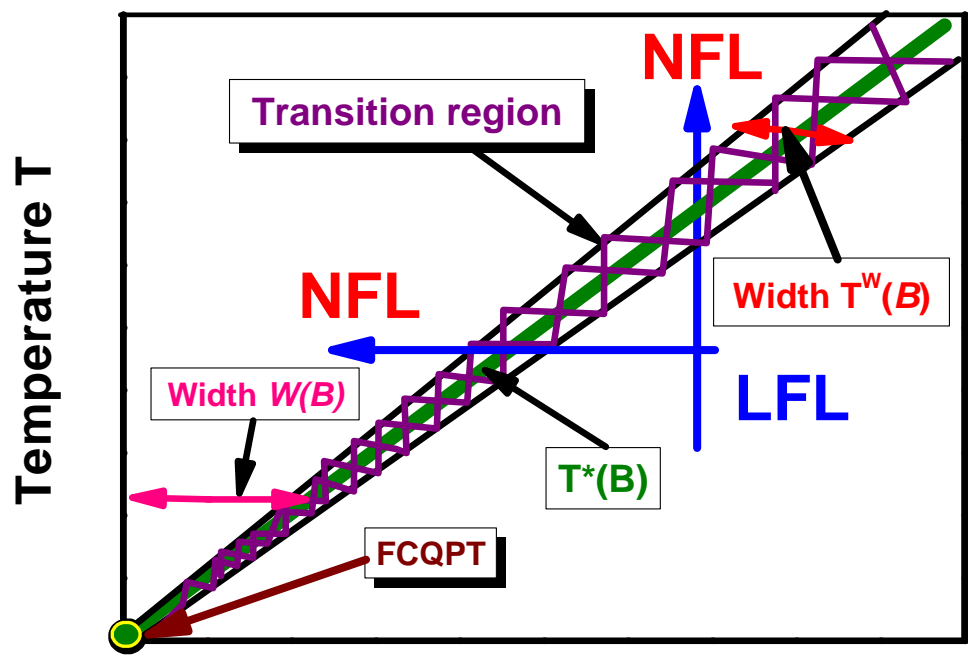

Magnetic field B

Fig. 1 (Color online). Schematic $T-B$ phase diagram of SCQSL with magnetic field as the control parameter. The vertical and horizontal arrows show LFL-NFL and NFL-LFL transitions at fixed $B$ and $T$, respectively. The dependences of the effective mass $M^{*}$ on $T$ and $B$ are given by Eqs. (4) and (5), respectively. The hatched area represents the transition region taking place at $T^{*}(B)$, see Eq. (8). The solid line in the hatched area represents the function $T^{*}(B) \simeq T_{M}(B)$ given by Eq. (7). The functions $W(B) \propto T \propto T^{*}$ and $T^{W}(B) \propto T \propto T^{*}$ shown by two-headed arrows define the width of the NFL state and the transition area, respectively. At FCQPT indicated by the arrow the effective mass $M^{*}$ diverges and both $W(B)$ and $T^{W}(B)$ tend to zero.

defined by the function $T^{*} \propto \mu_{B} B$, and the width $W(B)$ of the NFL state is seen to be proportional to $T$. In the same way, it can be shown that the width $T^{W}(B)$ of the transition region is also proportional to temperature [6].

\section{Dynamic spin susceptibility and low-frequency optical conductivity}

Now we turn to the neutron-scattering measurements of the dynamic spin susceptibility $\chi(\mathbf{q}, \omega, T)=\chi^{\prime}(\mathbf{q}, \omega, T)+i \chi^{\prime \prime}(\mathbf{q}, \omega, T)$ as a function of momentum $\mathbf{q}$, frequency $\omega$, and temperature $T$, former playing a crucial role in identifying the properties of the quasiparticle excitations involved. At low temperatures, such measurements reveal that the corresponding quasiparticles (belonging to herbertsmithite - a new type of insulator) are represented by spinons, form a continuum, and populate an approximately flat band crossing the Fermi level [5]. The imaginary part $\chi^{\prime \prime}\left(T, \omega_{1}\right)$ satisfies the equation $[8,14]$

$$
T^{2 / 3} \chi^{\prime \prime}\left(T, \omega_{1}\right) \simeq \frac{a_{1} \omega_{1}}{1+a_{2} \omega_{1}^{2}},
$$




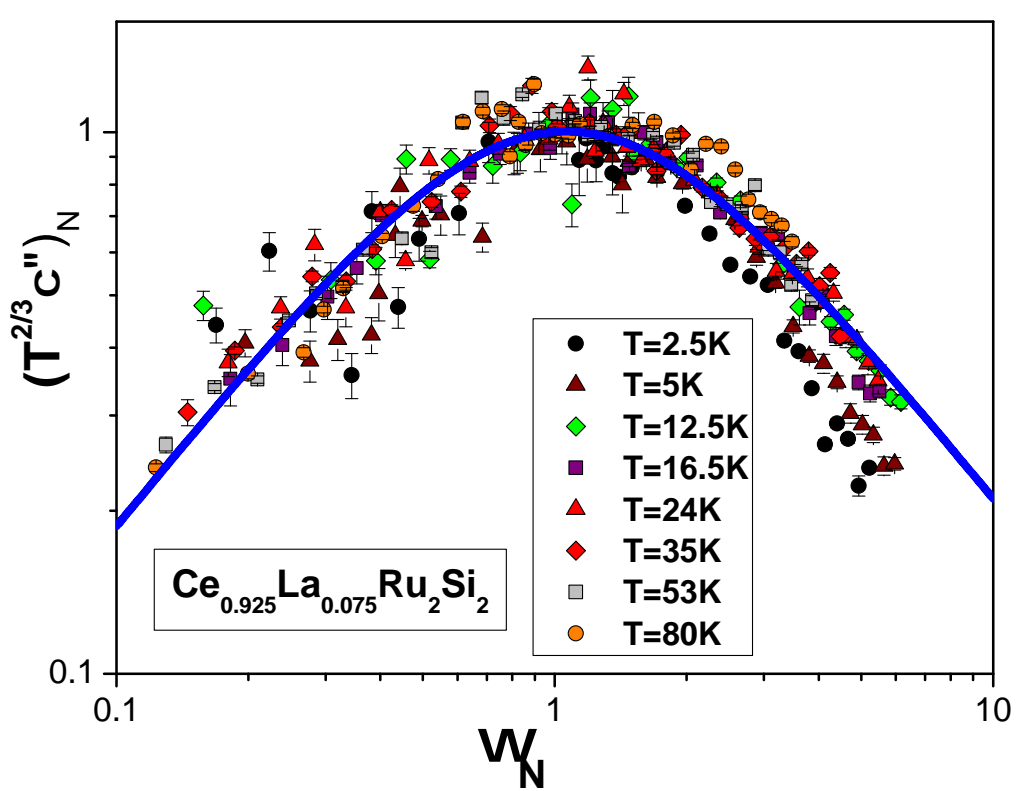

Fig. 2 (Color online) Scaling behavior of the normalized dynamic spin susceptibility $\left(T^{2 / 3} \chi^{\prime \prime}\right)_{N}$. Data are extracted from measurements on the heavy-fermion metal $\mathrm{Ce}_{0.925} \mathrm{La}_{0.075} \mathrm{Ru}_{2} \mathrm{Si}_{2}$ [25] and plotted against the dimensionless variable $E_{N}$. Solid curve: Theoretical calculations based on Eq. (10) [8].

where $a_{1}$ and $a_{2}$ are constants and $\omega_{1}=\omega /(T)^{2 / 3}$. It is seen from Eq. (9) that $T^{2 / 3} \chi^{\prime \prime}\left(T, \omega_{1}\right)$ has a maximum $\left(T^{2 / 3} \chi^{\prime \prime}\left(T, \omega_{1}\right)\right)_{\max }$ at some $\omega_{\max }$ and depends on the only variable $\omega_{1}$. Equation (9) confirms the scaling behavior of $\chi^{\prime \prime} T^{0.66}$ experimentally established in Ref. [4]. As it was done for the effective mass when constructing (6), we introduce the dimensionless function $\left(T^{2 / 3} \chi^{\prime \prime}\right)_{N}=$ $T^{2 / 3} \chi^{\prime \prime} /\left(T^{2 / 3} \chi^{\prime \prime}\right)_{\max }$ and the dimensionless variable $\omega_{N}=\omega_{1} / \omega_{\max }$, and Eq. (9) is modified to read

$$
\left(T^{2 / 3} \chi^{\prime \prime}\right)_{N} \simeq \frac{b_{1} \omega_{N}}{1+b_{2} \omega_{N}^{2}},
$$

with $b_{1}$ and $b_{2}$ are fitting parameters which are to adjust the function on the right hand side of Eq. (10) to reach its maximum value 1 at $\omega_{n}=1$. In such a situation it is expected that the dimensionless normalized susceptibility $\left(T^{2 / 3} \chi^{\prime \prime}\right)_{N}=$ $T^{2 / 3} \chi^{\prime \prime} /\left(T^{2 / 3} \chi^{\prime \prime}\right)_{\max }$ exhibits scaling as a function of the dimensionless energy variable $\omega_{N}[8,14]$. We predict that if measurements of $\chi^{\prime \prime}$ are taken at fixed $T$ as a function of $B$, then taking into account Eq. (5), we again obtain that the function 


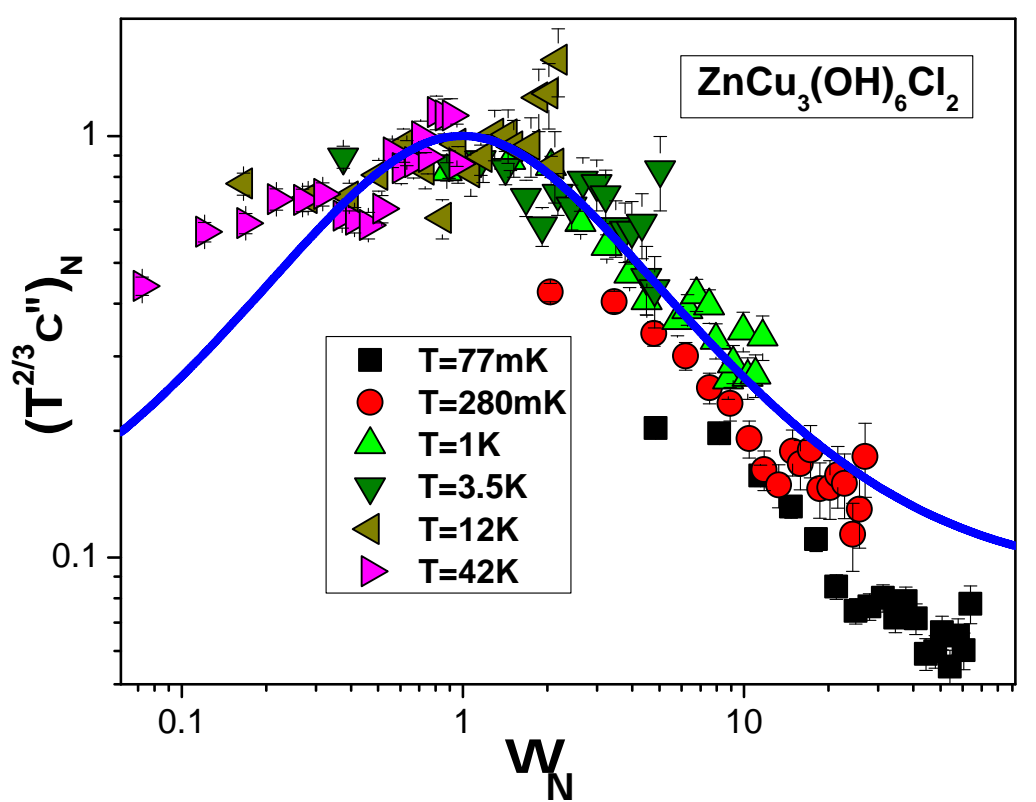

Fig. 3 (Color online) Scaling behavior of the normalized dynamic spin susceptibility $\left(T^{2 / 3} \chi^{\prime \prime}\right)_{N}$. Data are extracted from measurements on herbertsmithite $\mathrm{ZnCu}_{3}(\mathrm{OH})_{6} \mathrm{Cl}_{2}$ [4]. Solid curve: Theoretical calculations based on Eq. (10) [8].

$B^{2 / 3} \chi^{\prime \prime}(\omega)$ exhibits the scaling behavior with $\omega_{N}=\omega_{1} / \omega_{\max }$

$$
\left(B^{2 / 3} \chi^{\prime \prime}\right)_{N} \simeq \frac{d_{1} \omega_{N}}{1+d_{2} \omega_{N}^{2}},
$$

In the same way, $d_{1}$ and $d_{2}$ are fitting parameters adjusted such that the function $\left(B^{2 / 3} \chi^{\prime \prime}\right)_{N}$ reaches unity at $\omega_{N}=1$. If the system is exactly at a FCQPT point, the above scaling is valid down to lowest temperatures. Figure 2 displays values of $\left(T^{2 / 3} \chi^{\prime \prime}\right)_{N}$ extracted from measurements of the inelastic neutron-scattering spectrum on the heavy-fermion metal $\mathrm{Ce}_{0.925} \mathrm{La}_{0.075} \mathrm{Ru}_{2} \mathrm{Si}_{2}$ [25]. The scaled data for this quantity, obtained from the measurements on another quite different strongly correlated system such as $\mathrm{ZnCu}_{3}(\mathrm{OH})_{6} \mathrm{Cl}_{2}$ [4], are displayed in Fig. 3. It is seen that the theoretical results from Ref. [8] (solid curves) are in good agreement with the experimental data collected on all two compounds, for $\left(T^{2 / 3} \chi^{\prime \prime}\right)_{N}$ does exhibit the anticipated scaling behavior for these systems. The scaled data obtained in measurements on such quite different strongly correlated systems as $\mathrm{ZnCu}_{3}(\mathrm{OH})_{6} \mathrm{Cl}_{2}$ and $\mathrm{Ce}_{0.925} \mathrm{La}_{0.075} \mathrm{Ru}_{2} \mathrm{Si}_{2}$ collapse fairly well onto a single curve over almost three decades of the scaled variables.

Now we are in a position to consider the low-frequency optical conductivity $\bar{\sigma}$ of the insulator herbertsmithite. We focus on low temperatures $T$ and frequencies 
$\omega$, since the phonon absorption is expected to contaminate the conductivity at elevated $T$ and $\omega$ [12]. In the atomic units $\hbar=c=1$, the Hamiltonian of a particle with a spin reads

$$
\hat{H}=\frac{1}{2 m}(\mathbf{p}-e \mathbf{A})^{2}+e \phi-\frac{\mu_{B}}{s} \mathbf{s B}
$$

where $\mathbf{s}$ is the spin, $\mathbf{A}$ and $\phi$ are, respectively, the vector and scalar potentials. Also, $\mathbf{p}=i \nabla$ is the momentum operator of a particle, $e$ is its charge. We use the gauge $\nabla \mathbf{A}=0$, so that $\mathbf{p}$ and $\mathbf{A}$ commute. In case of spinons $e=0$, and therefore only the last term on the right hand side of Eq. (12) contributes to $\bar{\sigma}$.

As it follows from Eqs. (4) and (9), at low frequencies $\omega$ the imaginary part of the spin susceptibility is given by [8]

$$
\chi^{\prime \prime}(\omega) \propto \omega\left(M^{*}\right)^{2} .
$$

Taking into account that the energy transfer $\varepsilon_{B}$ [22] from the magnetic field $B(\omega)$ to the system is defined by the term $\left(\mu_{B} / s\right) \mathbf{s B}$ of Eq. (12), we obtain

$$
\varepsilon_{B}=2 \pi \omega\left[\frac{\mu_{B}}{s} \mathbf{s B}\right] \chi^{\prime \prime}(\omega) \equiv 2 \pi \frac{\omega^{2} \mu_{B}^{3}}{s}\left(M^{*}\right)^{2} \mathbf{s B}
$$

On the other hand, the energy transfer $\varepsilon_{E}$ from the electric field $E(\omega)$ is given by

$$
\varepsilon_{E}=E^{2}(\omega) \bar{\sigma}(\omega)
$$

Comparison of Eqs. (14) and (15) yields

$$
\bar{\sigma}(\omega) \propto \omega \chi^{\prime \prime}(\omega) \propto \omega^{2}\left(M^{*}\right)^{2}
$$

From Eq. (16) it follows that $\bar{\sigma}(\omega) \propto \omega^{2}$. We note that employed $\chi$ " given by Eq. (13) coincides at low $\omega$ with that given by Eq. (9), and gives a good description the facts, see see Figs. 2 and 3. At elevated temperatures it is seen from Eqs. (4) and (16) the low-frequency optical conductivity is a decreasing function of $T$. This observation is consistent with the experimental data [12]. It also follows from Eqs. (5) and (16) that $\bar{\sigma}(\omega)$ diminishes under the application of magnetic fields. Recent observation seems to contradict the experimental results since no systematic magnetic field dependence is observed [12]. To elucidate the magnetic field dependence of $\bar{\sigma}(B)$, we note that measurements of $\bar{\sigma}(B)$ have been taken at $6 \mathrm{~K}$ and the magnetic fields $B \leq 7 \mathrm{~T}$ [12]. As it is seen from Eq. (7) and Fig. 1, in such a case the system is still in the transition regime and does not enter into the LFL state at which the effective mass $M^{*}$ is given by Eq. (5). Therefore, in this case the effective mass behavior is determined by Eq. (4), rather than by Eq. (5), and the $\bar{\sigma}(B)$ dependence cannot be observed. As a result, we predict that the $B$-dependence of $\bar{\sigma}$ can be observed at $B \simeq 7 \mathrm{~T}$ provided that $T \leq 1 \mathrm{~K}$. 


\section{Conclusions}

In summary, based on recent measurements of the low-frequency optical conductivity in the herbertsmithite $\mathrm{ZnCu}_{3}(\mathrm{OH})_{6} \mathrm{Cl}_{2}$, we have shown that it can be viewed as a strongly correlated Fermi system whose properties is defined by SCQSL formed by chargeless spinons. Combining analytical arguments and those based entirely on experimental data analysis, we have come to conclusion that above quantum spin liquid forms a new state of matter represented by fermion condensate and discussed by us earlier (see, Refs. [6, 11, 14] for details). The crux of the matter here is that geometrical frustration generates almost dispersionless branches of the quasiparticles (spinons in this case) spectrum (so-called flat bands), which makes the system prone to ordinary LFL instability related to the quasiparticle effective mass divergence $[6,14]$. This instability, in turn, yields the fermion condensate - the new state of matter, emerging during FCQPT. To analyze the experimental manifestations of the above new state of matter in herbertsmithite, we consider the dynamic spin susceptibility and calculate the lowfrequency optical conductivity of 2D spin-carrying insulators with geometrical frustration. For that we employ the model of strongly correlated quantum spin liquid located near the FCQPT. The comparison of our theoretical results with experimental data on the herbertsmithite low-frequency optical conductivity allows us to reveal its temperature dependence. We also have predicted a dependence of the optical conductivity on magnetic fields and pointed out explicitly conditions at which such dependence can be experimentally observed. Our findings not only establish the existence of the robust SCQSL state in herbertsmithite, but also provide a strategy for analyzing this state in insulators of new type.

Acknowledgements We are grateful to V.A. Khodel for valuable discussions. This work was partly supported by U.S. DOE, Division of Chemical Sciences, Office of Basic Energy Sciences, Office of Energy Research.

\section{References}

1. J.S. Helton, K. Matan, M.P. Shores, E.A. Nytko, B.M. Bartlett, Y. Yoshida, Y. Takano, A. Suslov, Y. Qiu, J.-H. Chung, D.G. Nocera, Y.S. Lee, Phys. Rev. Lett. 98, 107204 (2007)

2. T.H. Han, J.S. Helton, S. Chu, A. Prodi, D.K. Singh, C. Mazzoli, P. Müller, D.G. Nocera, Y.S. Lee, Phys. Rev. B 83, 100402(R) (2011)

3. M. A. deVries, K.V. Kamenev, W.A. Kockelmann, J. Sanchez-Benitez, A. Harrison Phys. Rev. Lett. 100, 157205 (2008)

4. J.S. Helton, K. Matan, M.P. Shores, E.A. Nytko, B.M. Bartlett, Y. Qiu, D.G. Nocera, Y.S. Lee, Phys. Rev. Lett. 104, 147201 (2010)

5. T.H. Han, J.S. Helton, S. Chu, D.G. Nocera, J.A. Rodriguez-Rivera, C. Broholm, Y.S. Lee, Nature 492, 406 (2012)

6. V. R. Shaginyan, M. Ya. Amusia, A. Z. Msezane, K. G. Popov, Phys. Rep. 492, 31 (2010)

7. V.R. Shaginyan, A.Z. Msezane, K.G. Popov, Phys. Rev. B 84, 060401(R) (2011) 
8. V.R. Shaginyan, A.Z. Msezane, K.G. Popov, V.A. Khodel, Phys. Lett. A 376, $2622(2012)$

9. V.R. Shaginyan, A.Z. Msezane, K.G. Popov, G.S. Japaridze, V.A. Stephanovich, Europhys. Lett. 97, 56001 (2012)

10. H.J. Liao, Z.Y. Xie, J. Chen, Z.Y. Liu, H.D. Xie, R.Z. Huang, B. Normand, T. Xiang, Phys. Rev. Lett. 118, 137202 (2017)

11. V.R. Shaginyan, V.A. Stephanovich, A.Z. Msezane, P. Schuck, J.W. Clark, M. Ya. Amusia, G.S. Japaridze, K.G.Popov, E.V. Kirichenko, J. Low Temp. Phys. (2017), https://doi.org/10.1007/s10909-017-1801-3

12. D.V. Pilon, C.H. Lui, T.-H. Han, D. Shrekenhamer, A.J. Frenzel, W.J. Padilla, Y.S. Lee, N. Gedik, Phys. Rev. Lett. 111, 127401 (2013)

13. A.C. Potter, T. Senthil, P.A. Lee, Phys. Rev. B 87, 245106 (2013)

14. M. Ya. Amusia, K. G. Popov, V. R. Shaginyan, V. A. Stephanovich, Theory of Heavy-Fermion Compounds, Springer Series in Solid-State Sciences, 182, Springer, Heidelberg, New York, Dordrecht, London, 360 p. (2015)

15. V. A. Khodel, V.R. Shaginyan, JETP Lett. 51, 553 (1990)

16. D. Green, L. Santos, C. Chamon, Phys. Rev. B 82,075104 2010

17. T.T. Heikkilä, N.B. Kopnin, G.E. Volovik, JETP Lett. 94, 2332011

18. G.E. Volovik, JETP Lett. 53, 222 (1991)

19. G.E. Volovik, in Quantum analogues: From Phase Transitions to Black Holes and Cosmology, eds. W.G. Unruh, R. Schutzhold. Springer Lecture Notes in Physics, vol. 718 (Springer, Orlando, 2007), p.31

20. L.D. Landau, Sov. Phys. JETP 3, 920 (1956)

21. E.M. Lifshitz, L.P. Pitaevskii, Statisticheskaya Fizika (Statistical Physics), Pt. 2, Nauka, Moscow, 1978; Translated into English, Pergamon Press, Oxford, 1980

22. D. Pines, P. Noziéres, Theory of Quantum Liquids, Benjamin, New York, 1966

23. J.W. Clark, V.A. Khodel, M.V. Zverev, Phys. Rev. B 71, 012401 (2005)

24. V.R. Shaginyan, K.G. Popov, V.A. Stephanovich, V.I. Fomichev, E.V. Kirichenko, Europhys. Lett. 93, 17008 (2011)

25. W. Knafo, S. Raymond, J. Flouquet, B. Fåk, M.A. Adams, P. Haen, F. Lapierre, S. Yates, P. Lejay, Phys. Rev. B 70, 174401 (2004) 